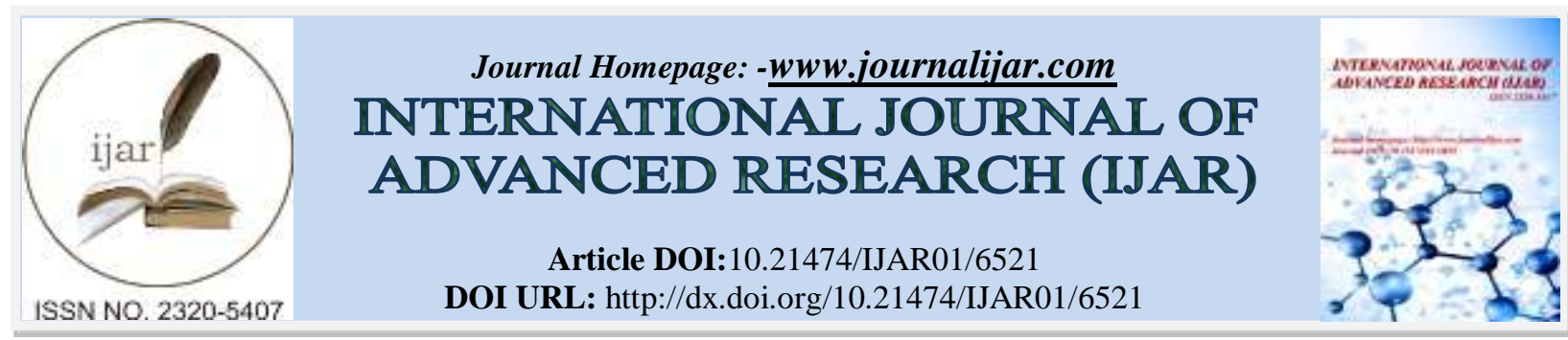

RESEARCH ARTICLE

\title{
AN ENHANCEMENT REVIEW ON IMAGE SEGMENTATION METHODS.
}

\section{R.V.Santhi ${ }^{1}$ and E. Ramaraj ${ }^{2}$.}

1. Assistant Professor, Department of Computer Science, S. T. Hindu College, Nagercoil.

2. Professor, Department of Computer Science \& Engineering, Alagappa University, Karaikudi.

\section{Manuscript Info}

Manuscript History

Received: 13 December 2017

Final Accepted: 15 January 2018

Published: February 2018

Key words:-

Segmentation, Natural Image,

Medical Image, Satellite Image.

\begin{abstract}
Image segmentation is one of the chief issues in image processing functions and it is challenged by intensity, variation in images and noises. The researchers are continued on the image segmentation way to induce the performance of other image processing tasks because image segmentation is a preprocessing entity to the pair the image to produce further refined result. The research people are confused on which method is better for which image type. The major image types considered in segmentation fields are natural image and satellite image. This paper provides on comparative study on the recent development of image segmentation papers to understand the behavior and perceptual characteristics owned by them. The analysis carried out through the parameters such as processing method name, accuracy, advantages and disadvantages. This study can be much useful for young researchers who are under the research umbrella of image segmentation.
\end{abstract}

Copy Right, IJAR, 2018,. All rights reserved.

\section{Introduction:-}

Image segmentation is an important issue of many image processing tasks which can be used as a preprocessing job. Image segmentation segments or groups the similar contents of an image to achieve the separation of multiple or single objects. Image segmentation is configured by either supervised or unsupervised. It divided the image into meaningful parts and that parts can be contributed to the processing of three type of images such as natural, medical and satellite images. Segmentation plays a main role in human detection, bird detection and animal detection. The medical image segmentation is used to analyze the human scanned organs to assist the pathologists to plan an operation or treatment. The satellite image segmentation is carried out to help the segmentation of space objects to aid the galaxy study. The researchers need to understand the behavior of recent segmentation methods and the usage of them. So, that this paper makes a study of recently published image segmentation papers.

\section{Literature Review:-}

DeepikaKoundal [1] proposes a method to transforms the image into the Neutrosophic domain and then extracts the texture features using analogies of human retentive texture discrimination mechanisms. The Neutrosophic clustering is employed to segment the images. This method can handle the indeterminacy of pixels to have strong clusters and to perform segmentation effectively with the noisy images. The evaluation of this paper with other segmentation methods is to measure its performance which shows its robustness for noisy and textural images.

Mohammad HamedMozaffari [2] et al. utilizes convergence heterogeneous particle swarm optimization (PSO) algorithm to find the optimal multilevel thresholds. The general idea of this algorithm is to divide particles into four 
sub swarms for searching problem space. Otsu's and Kapur'sthresholding methods are separately used as fitness function which the former maximize between-class variance and the latter maximize image entropy. To evaluate this paper, it applied to a benchmark of images and the results compared with similar and famous heuristic methods, genetic algorithm, harmony search and the PSO.

Ya'nan Zhou [3] et al. presents an adaptive approach for defining and estimating the optimal scale in the multiscale segmentation process. Central to this method is the combined use of image features from segmented objects and prior knowledge from historical thematic maps in a top-down segmentation procedure. To apply this method on a GF-1 multispectral satellite image and a ZY-3 multispectral satellite image to produce multiscale segmentation maps and further classification maps, compared with the state-of-the art and the traditional mean shift algorithm.

JianbingShen [4] et al. proposes a novel energy minimization method for general higher-order binary energy functions. In this, first relax a discrete higher-order function to a continuous one, and use the Taylor expansion to obtain an approximate lower-order function, which is optimized by the quadratic pseudo-Boolean optimization (QPBO) or other discrete optimizations. The minimum solution of this lower-order function is then used as a new local point, where we expand the original higher-order energy function again. This algorithm does not restrict to any specific form of the higher-order binary function or bring in extra auxiliary variables.

Ankayarkanni [5] et al. develops a technique that can detect changes in urban buildings after natural disasters such as earthquakes, typhoons or tsunamis. This is also helpful to providing accurate information about the changes for urban planning and updating Geo-spatial information system (GIS). It is necessary that the remote sensing imagery has to be converted into some meaning information, which in turn requires some segmentation methods followed by classification. This paper proposes few object based method (K-Means, KFCM, and Moving KFCM) for classification that segments the image followed by classification.

Ganesan et al. [15] presents a novel approach for the segmentation of high resolution satellite images using the spatial information incorporated modified fuzzy C-means clustering algorithm. The images after preprocessing and geo referencing, they are available in RG B color space. RGB color space is not widely used for segmentation and pattern recognition. In this paper, this problem can be solved by the application of perceptually uniform color spaces like CIELab or CIELuv. The FCM algorithm is one of the efficient approaches for image segmentation; it doesn't give any spatial information which is important for clustering problems. In modified FCM clustering algorithm, the spatial information is incorporated as a function of the weighted sum of the membership function. As compared to the result of other methods, this paper is robust and efficient for the segmentation of satellite Images.

Sathya et al. [6] describes the implementation of two algorithms, namely Back Propagation algorithm of ANN and K-Means algorithm on wide database of images. It provides the tool for segmentation and classification of remote sensing images. This classified image is given to K-Means algorithm and Back Propagation algorithm of ANN to calculate the density count. The density count is stored in database for future reference and for other applications. This paper has the capability to show comparison results of both algorithms. K-Means algorithm gives very high accuracy, but it is used only in single database. Neural Network is useful for multiple databases and also provides good accuracy.

Ana Carolina QuinlaoSiravenha [7] et al.describes a novel approach based on Discrete Cosine Transformation (DCT) and compared it with some approaches based on statistical metrics. Both, the DCT and statistical approaches, make a texture analysis based on components of gray scale satellite image from QuickBird QB2 and WordView WV02 sensors. The texture analysis is based on the similarity property within a group of pixels that allows simple sub-blocks manipulation. The DCT is applied for the image and the AC energy component is calculated individually for square blocks. In this paper, segmentation rules are defined based on those values of energy. The statistical metrics adopted for comparison are the mean, standard deviation, median, and variance.

SayleeGharge et al. [8] propose a new algorithm for SAR image segmentation using vector quantization technique on entropy image. Initially, this paper obtain entropy image and in second step it uses Kekre's Fast Codebook Generation (KFCG) algorithm for segmentation of the entropy image. Thereafter, a codebook of size 128 is generated for the entropy image. These code vectors are further clustered into 8 clusters using same KFCG algorithm and converted into 8 images. These 8 images are displayed as a result. This approach does not lead to over segmentation or under segmentation. This approach gives better segmentation with less complexity. 
Salem Saleh Al-amri et al. [9] presents methods for edge segmentation of satellite images. This paper uses seven techniques such as Sobel operator technique, Prewitt technique, Kiresh technique, Laplaciantechnique, Canny technique, Roberts technique and Edge maximization technique (EMT) and they are compared with one another so as to choose the best technique for edge detection segment image. These techniques applied on one satellite images to choose base guesses for segmentation or edge detection image.

Ramadevi et al. [10] discusses the interaction between image segmentation and object recognition. Edge detection methods such as Sobel, Prewitt, Roberts, Canny, Laplacian of Guassian (LOG) are used for segmenting the image. Expectation-Maximization (EM) algorithm, OSTU and Genetic algorithms are used to demonstrate the synergy between the segmented images and object recognition. MATLAB 7.9 is used for experimentation image. EM algorithm and OSTU algorithm exhibited stable segmentation effect.

Ming Shao et al. [11] propose a super resolution based method to enhance the quality of near infrared face image using tensor face. It is organized as follows: 1) introduces the relationship between NIR and VIS images and reveals the essence of their different appearances. 2) Using tensor face as a basic multi linear analysis tool to organize image ensembles and then present an algorithm aimed at obtaining the high resolution identity by super-resolution. 3) The image fusion technique to compensate low frequency components of simulated images. Finally the result is promising and the fused image is proved to be more suitable for human to recognize or verify.

Te-Won Lee et al. [12] derives an unsupervised classification algorithm by modeling observed data as a mixture of several mutually exclusive classes that are each described by linear combinations of independent, non-Gaussian densities. This improves classification accuracy compared with standard Gaussian mixture models. To apply this technique, to the problem of unsupervised classification segmentation and denoising of images is also useful for denoising and filling in missing pixels in images with complex structures. The advantage of this model is that image codes can be learned with increasing numbers of classes thus providing greater flexibility than in either Gaussian mixture model or standard independent component analysis; (ICA) algorithms.

Charles H. Fosgate et al. [13] presents efficient multi scale approaches to the segmentation of natural clutter, specifically grass and forest, and to the enhancement of anomalies in synthetic aperture radar (SAR) imagery. This paper employ recently introduced classes of multi scale stochastic processes that provide a powerful framework for describing random processes and fields that evolve in scale. This build models representative of each category of terrain of interest and employ them in directing decisions on pixel classification, segmentation, and anomalous behavior. Anomaly enhancement is possible with minimal additional computation.

Leonardo Sant' Anna Bins et al. [14] presents a segmentation method based on a region growing approach. It is implemented in the geographic information and image processing system (SPRIN G) which is developed at INPE. Segmentation of satellite images is very complex. A simple segmentation scheme based on region growing approach is applied to segment images which are being used to access land use changes in the Amazon region.

\section{Discussion:-}

The comparative nature of the research papers listed in the Table 1. The natural image segmentation is achieved through the papers Higher-order energies for image segmentation. The paper produces higher segmentation accuracy for natural image segmentation. The advantage of this paper is used to exhibit the state segmentation effect. The major method used in this category QPBO.

The satellite image segmentation is carried out through the research methods. The best result provides for this case is Adaptive Approach Optimal code method. The major method used in this category is enhance the quality of near infrared images. .

Table 1:- Analysis on segmentation methods

\begin{tabular}{|l|l|l|l|l|l|}
\hline Author & $\begin{array}{l}\text { Reference } \\
\text { Paper }\end{array}$ & Year & Publisher & $\begin{array}{l}\text { Segmentation } \\
\text { method used }\end{array}$ & Image type \\
\hline DeepikaKoundal & {$[1]$} & 2017 & IET & PSO & Satellite \\
\hline $\begin{array}{l}\text { Mohammad } \\
\text { HamedMozaffari et al. }\end{array}$ & {$[2]$} & 2017 & IET & Neutrosophic & Natural \\
\hline
\end{tabular}




\begin{tabular}{|l|l|l|l|l|l|}
\hline Ya'nan Zhou et al. & {$[3]$} & 2017 & IEEE & $\begin{array}{l}\text { Adoptive Approach } \\
\text { Optimal Scale }\end{array}$ & Satellite \\
\hline JianbingShen et al. & {$[4]$} & 2017 & IEEE & QPSO & Natural \\
\hline B. Ankayarkanni et al. & {$[5]$} & 2014 & IJCSE & $\begin{array}{l}\text { Object faced } \\
\text { method }\end{array}$ & Satellite \\
\hline Ganesan P et al. & {$[15]$} & 2014 & MAGNT & Modified FCM & Satellite \\
\hline P. Sathya et al. & {$[6]$} & 2011 & IJMLC & BPN \& K-means & Remote Sensing \\
\hline $\begin{array}{l}\text { Ana Carolina } \\
\text { QuintaoSiravenha et } \\
\text { al. }\end{array}$ & {$[7]$} & 2011 & INPE & $\begin{array}{l}\text { Proposed } \\
\text { Segmentation } \\
\text { Algorithm }\end{array}$ & Satellite \\
\hline SayleeGharge et al. & {$[8]$} & 2011 & IJCSIS & KFCG & Satellite \\
\hline $\begin{array}{l}\text { Salem Saleh Al-amri } \\
\text { et al. }\end{array}$ & {$[9]$} & 2010 & IJCSE & $\begin{array}{l}\text { Canny edge based } \\
\text { segmentation }\end{array}$ & Satellite \\
\hline $\begin{array}{l}\text { Y. Ramadevi et al. } \\
\text { Ming Shao et al. }\end{array}$ & {$[10]$} & 2010 & IJCSIT & $\begin{array}{l}\text { Genetic Based } \\
\text { Segmentation }\end{array}$ & Natural \\
\hline Te-Won Lee et al. & {$[12]$} & 2009 & IEEE & Super Resolution & Natural \\
\hline $\begin{array}{l}\text { Charles H. Fosgate et } \\
\text { al. }\end{array}$ & {$[13]$} & 1997 & IEEE & ICA & Natural \\
\hline $\begin{array}{l}\text { Leonardo Sant Anna } \\
\text { Bins et al. }\end{array}$ & {$[14]$} & 1996 & INPE & $\begin{array}{l}\text { Multiscale } \\
\text { Stochastic process }\end{array}$ & Satellite \\
\hline
\end{tabular}

\section{Conclusion:-}

This paper analyses the recent publication in image segmentation to characterize the segmentation behaviors. The publications are analyzed through the author, year, title, publication, applied image types. This work declares that the highly accuracy method for natural image segmentation is QPBO method. For satellite images the best method is Adaptive Approach Optimal method. The prescribed segmentation methods provide a better study on forthcoming users which can enhance the fresh research fellow's thoughts on image processing. This paper can be further improved by adding more papers to extend this comparative analysis.

\section{References:-}

1. DeepikaKoundal, "Texture-based image segmentation using neutrosophic clustering”, IET Image Processing, Vol. 11, Issue 8, PP. 640-645, Jul 2017.

2. Mohammad HamedMozaffari and Won-Sook Lee, "Convergent heterogeneous particle swarm optimization algorithm for multilevel image thresholding segmentation”, IET Image Processing, Vol.11, Issue 8, PP. 605619, Aug 2017.

3. Ya'nan Zhou, Jun Li, Li Feng, Xin Zhang, and Xiaodong Hu, "Adaptive scale selection for multiscale segmentation of satellite images", IEEE Journal of Selected Topics in Applied Earth Observations and Remote Sensing, Vol. 10, Issue 8, Aug 2017.

4. JianbingShen, JiantengPeng, Xingping Dong, Ling Shao, and FatihPorikli, "Higher-order energies for image segmentation", IEEE Transactions on Image Processing, Vol.26, Issue 10, Oct 2017.

5. B. Ankayarkanni and Ezil Sam Leni, "Object based segmentation techniques for classification of satellite image", Indian Journal of Computer Science and Engineering, Vol. 5, No. 3, Jan-Jul 2014.

6. P. Sathya and L. Malathi, "Classification and segmentation in satellite imagery using Back Propagation algorithm of ANN and K-Means algorithm", International Journal of Machine Learning and Computing, Vol. 1, No. 4, Oct 2011.

7. Ana Carolina QuintaoSiravenha and EvaldoGolcalvesPelaes, "The use of discrete cosine transform for satellite images segmentation and comparison to statistical metrics", Anasis XV SimposioBrasileiro de SensoriamentoRemoto-SBSR, Curitiba, PR, Brasil, INPE, P. 7271, 30 April-5 May,2011.

8. SayleeGharge, Tanuja K. Sarode and H.B. Kekre, "SAR image segmentation using vector quantization technique on entropy images", Ingernational Journal of Computer Science and Information Security, Vol. 7, No. 3, March 2010.

9. Salem Saleh Al-amri, N.V. Kalyankar and Khamitkar S.D, "Image segmentation by using edge detection", IJCSE, Vol. 02, No. 03, 2010. 
10. Y. Ramadevi, T. Sridevi, B. Poornima and B. Kalyani, "Segmentation and Object recognition using edge detection techniques", International Journal of Computer Science \& Information Technology, Vol. 2, No. 6, Dec 2010.

11. Ming Shao, Yumhong Wang and Yiding Wang, "A super-resolution based method to synthesize visual images from near infrared", IEEE ICIP, 2009.

12. Te-Won Lee and Michael S. Lewicki, "Unsupervised image classification, segmentation, and enhancement using ICA mixture models", IEEE Xplore, 2002.

13. Charles H. Fosgate, Hamid Krim, William W. Irving, William C. Kari and Alan S. Willsky, "Multiscale segmentation and anomaly enhancement of SAR imagery", IEEE Transactions on Image Processing, Vol. 6, No. 1, Jan 1997.

14. Leonardo Sant Anna Bins, Leila M. Garcia Fonseca, Guaraci Jose Erthal and Fernando Mitsuo Ii, "Satellite imagery segmentation: a region growing approach", Anais VIII SimposioBrasileiro de SensoriamentoRemoto, Salvador, Brasil, INPE, P. 677-680, 14-19 April 1996.

15. Ganesan P, V. Rajini, B.S. Sathish and KhamarBashaShaik, "CIELAB color space based high resolution satellite image segmentation using modified fuzzy C-Means clustering”, MAGNT Research Report, Vol. 2, Issue 6, PP. 199-210. 